\title{
Lamb waves in multilayered media: 3D and 6D formalisms
}

\author{
Anna Avershyeva ${ }^{1}$ and Sergey Kuznetsov ${ }^{1,2,3^{*}}$ \\ ${ }^{1}$ Moscow State University of Civil Engineering, Yaroslavsckoe sh. 26, Moscow, Russia \\ ${ }^{2}$ Ishlinsky Institute for Problems in Mechanics RAS, Pr. Vernadskogo 101, Moscow, Russia \\ ${ }^{3}$ Bauman Moscow State Technical University, ${ }^{\text {nd }}$ Institutskaya 5, Moscow, Russia
}

\begin{abstract}
A mathematical model for analyzing Lamb waves propagating in stratified media with arbitrary elastic anisotropy is worked out. The model incorporates a combined Fundamental Matrix (FM) and Modified Transfer Matrix (MTM) methods. Multilayered unbounded plates with different types of boundary conditions imposed on the outer surfaces are considered. Closed form fundamental matrices and secular equations for dispersion relations are derived.
\end{abstract}

\section{Introduction}

The genuine Lamb waves [1] are surface acoustic waves propagating in an unbounded isotropic homogeneous plate subjected to traction-free boundary conditions at the outer planes:

The genuine Lamb waves [1] are surface acoustic waves propagating in an unbounded isotropic homogeneous plate subjected to traction-free boundary conditions at the outer planes:

$$
\left.\mathbf{t}_{\mathbf{v}}(\mathbf{x}, t) \equiv \mathbf{v} \cdot \mathbf{C} \cdot \cdot \nabla_{\mathbf{x}} \mathbf{u}(\mathbf{x}, t)\right|_{x^{\prime}=-h} ^{x^{\prime}=0}=0
$$

In (1.1) $\mathbf{t}_{\mathbf{v}}$ are surface tractions, $\mathbf{v}$ is the unit normal to one of the boundary planes, $\mathbf{C}$ is the fourth order elasticity tensor (in the original Lamb's analysis tensor $\mathbf{C}$ was isotropic), $\mathbf{u}$ is the displacement field, $t$ is time,

$$
x^{\prime}=\mathbf{x} \cdot \mathbf{v}
$$

is the coordinate normal to the plane $\mathbf{x} \cdot \boldsymbol{v}=0$, and $h$ is the depth of a plate; see, Fig.1, where $\mathbf{n}$ is the unit vector normal to the wave front.

We shall also consider clamped boundary planes:

$$
\left.\mathbf{u}(\mathbf{x}, t)\right|_{x^{\prime}=-h} ^{x^{\prime}=0}=0
$$

and mixed boundary conditions for the traction-free upper plane and clamped bottom plane:

\footnotetext{
* Corresponding author: kuzn-sergey@yandex.ru
} 


$$
\begin{aligned}
& \left.\mathbf{t}_{\mathbf{v}}(\mathbf{x}, t)\right|^{x^{\prime}=0}=0 \\
& \left.\mathbf{u}(\mathbf{x}, t)\right|_{x^{\prime}=-h}=0
\end{aligned} .
$$

For a multilayered plate, the interface boundary conditions are conditions of the ideal mechanical contact:

$$
\begin{aligned}
& \left.\mathbf{t}_{\mathbf{v}}^{(n)}(\mathbf{x}, t)\right|_{x^{\prime}=-h_{n}}=\left.\mathbf{t}_{\mathbf{v}}^{(n+1)}(\mathbf{x}, t)\right|^{x^{\prime}=0} \\
& \left.\mathbf{u}^{(n)}(\mathbf{x}, t)\right|_{x^{\prime}=-h_{n}}=\left.\mathbf{u}^{(n+1)}(\mathbf{x}, t)\right|^{x^{\prime}=0},
\end{aligned}
$$

where index $n$ is referred to the corresponding layer. Equations (1.5) are written in the local coordinate systems with origins placed at the upper boundaries of the corresponding layers.

Regarding polarization (that is the direction of particle motion at the wave front), the genuine Lamb wave resemble Rayleigh wave [2], which propagate in a halfspace with the traction-free boundary condition at the boundary plane, but Lamb wave differ from Rayleigh wave in absence of necessity to impose Sommerfield's emission condition:

$$
\mathbf{u}(\mathbf{x}, t)=O\left(\left|x^{\prime}\right|^{-1}\right), \quad\left|x^{\prime}\right| \rightarrow \infty .
$$

Sommerfield's condition (1.6) is used to remove solutions for Rayleigh waves that do not attenuate with depth, as being physically unreasonable.

Generally, Lamb wave in a particular layer is sought in the form [3-6]:

$$
\mathbf{u}(\mathbf{x}, t)=\left(\sum_{k=1}^{6} C_{k} \mathbf{m}_{k} f_{k}\left(x^{\prime \prime}\right)\right) e^{i r(\mathbf{n} \cdot \mathbf{x}-c t)},
$$

where $C_{k}$ are the unknown complex coefficients defined up to a multiplier from the boundary or interface conditions $(1.1)-(1.5) ; \mathbf{m}_{k}$ are the unknown polarization vectors; $f_{k}$ are also unknown scalar complex-valued functions; the exponential multiplier $e^{i r(\mathbf{n} \cdot \mathbf{v}-c t)}$ corresponds to propagation of the plane wave front along direction $\mathbf{n}$ with the phase speed $c ; r$ is the wave number; dimensionless coordinate $x^{\prime \prime}$ in (1.7) is defined by

$$
x^{\prime \prime}=i r x^{\prime} .
$$

In most of the works on surface acoustic waves a problem of constructing the displacement field (1.7) is not considered. Instead, by satisfying all the boundary and interface conditions, the disperse relations between the phase speed $c$ and wave number $r$ are derived either analytically for single-layered plates $[7,8]$, or numerically for multilayered plates [9-13]. Quite often relations $c-r$ are replaced by relations $c-\omega$, where $\omega$ is the frequency:

$$
\omega=r c \text {. }
$$

However, even for a simpler problem of finding disperse relations, polarization vectors $\mathbf{m}_{k}$ and displacement distribution functions $f_{k}$ need generally to be constructed. Vectors $\mathbf{m}_{k}$ are either genuine or generalized eigenvectors of the Christoffel equation, introduced later. Analogously, functions $f_{k}$ are eigenfunctions of an ordinary differential equation, to which the Christoffel equation is the characteristic equation.

Herein, an alternative approach will be introduced, which removes necessity to create and analyze vectors $\mathbf{m}_{k}$ and functions $f_{k}$. This approach is based on constructing the fundamental matrix for a system of the first-order differential equations and finding eigensolutions for the Sturm-Liouville problem; see also $[14,15]$, where a method based on constructing the fundamental matrix and utilizing Stroh's six-dimensional formalism [16], 
was used for studying Flouqet's problem for periodically layered composite plates. In the present paper a different six-dimensional formalism developed in $[17,18]$ will be used. Applying this formalism will allow us to obtain closed form fundamental matrices and secular equations for Lamb waves propagating in multilayered plates with arbitrary elastic anisotropy.

\section{Governing equations}

All the regarded layers are assumed to be homogeneous, anisotropic, and linearly hyperelastic. Equation of motion for homogeneous anisotropic elastic medium can be written in the form:

$$
\mathbf{L}\left(\partial_{\mathbf{x}}, \partial_{t}\right) \mathbf{u} \equiv \operatorname{div}_{\mathbf{x}} \mathbf{C} \cdot \nabla_{\mathbf{x}} \mathbf{u}-\rho \ddot{\mathbf{u}}=0,
$$

where $\rho$ is the material density, and $\mathbf{C}$ is the elasticity tensor assumed to be positive definite:

$$
\underset{\mathbf{S} \in \operatorname{sym}\left(R^{3} \otimes R^{3}\right), \mathbf{A} \neq 0}{\forall \mathbf{S}}(\mathbf{S} \cdot \mathbf{C} \cdot \mathbf{S}) \equiv \sum_{i, j, m, n} S_{i j} C^{i j m n} S_{m n}>0 .
$$

Condition (2.2) ensures strong ellipticity of tensor $\mathbf{C}$ :

$$
\underset{\mathbf{a}, \mathbf{b} \in R^{3}, \mathbf{a}, \mathbf{b} \neq 0}{\forall \mathbf{a}, \mathbf{b}} \quad \mathbf{a} \otimes \mathbf{b} \cdot \mathbf{C} \cdot \mathbf{b} \otimes \mathbf{a}>0 .
$$

The latter condition is equivalent to positive definiteness of the acoustic tensor

$$
\mathbf{A}(\mathbf{b}) \equiv \mathbf{b} \cdot \mathbf{C} \cdot \mathbf{b}
$$

at any $\mathbf{b} \in R^{3}, \mathbf{b} \neq 0$.

\subsection{D-formalism}

Now, we outline a formal procedure for finding the unknown polarization vectors $\mathbf{m}_{k}$ and functions $f_{k}$. Substituting representation (1.7) into Eq. (2.1) yields a system of the secondorder matrix equations with respect to functions $\mathbf{m}_{k} f_{k}$ :

$$
\left(\mathbf{A}_{1} \partial_{x^{\prime \prime}}^{2}+\left(\mathbf{A}_{2}+\mathbf{A}_{2}^{t}\right) \partial_{x^{\prime \prime}}+\mathbf{A}_{3}\right) \cdot \mathbf{m}_{k} f_{k}=0
$$

where

$$
\mathbf{A}_{1}=\mathbf{v} \cdot \mathbf{C} \cdot \mathbf{v}, \quad \mathbf{A}_{2}=\mathbf{v} \cdot \mathbf{C} \cdot \mathbf{n}, \quad \mathbf{A}_{3}=\mathbf{n} \cdot \mathbf{C} \cdot \mathbf{n}-\rho c^{2} \mathbf{I} .
$$

Assuming functions $f_{k}$ to have Euler's exponential representation

$$
f_{k}\left(x^{\prime \prime}\right)=e^{i r \gamma_{k} x^{\prime}}
$$

with the unknown parameters $\gamma_{k}$ (these are sometimes called the Christoffel parameters) and substituting this representation into Eq. (2.5), we arrive at the Christoffel equation:

$$
\left(\mathbf{A}_{1} \gamma_{k}^{2}+\left(\mathbf{A}_{2}+\mathbf{A}_{2}{ }^{t}\right) \gamma_{k}+\mathbf{A}_{3}\right) \cdot \mathbf{m}_{k}=0
$$

The latter equation reveals that exponential parameters $\gamma_{k}$ are roots of a polynomial of the sixth degree with respect to $\gamma_{k}$ :

$$
\operatorname{det}\left(\mathbf{A}_{1} \gamma_{k}^{2}+\left(\mathbf{A}_{2}+\mathbf{A}_{2}{ }^{t}\right) \gamma_{k}+\mathbf{A}_{3}\right)=0
$$

On obtaining the Christoffel parameters from Eq. (2.9), we can find polarization vectors $\mathbf{m}_{k}$ as the eigenvectors of a matrix in the left-hand side of Eq. (2.8). 
Remark 2.1. If a matrix in the left-hand side of Eq. (2.8) has Jordan blocks (this case is known as the non-semisimple degeneracy), then representation (2.7) becomes insufficient. Within framework of both 3D and 6D-formalisms this case was studied in [19].

\section{2. $6 D$-formalism}

Now it will be demonstrated that the preceding stage of finding the Christoffel parameters $\gamma_{k}$ and polarization vectors $\mathbf{m}_{k}$ can be avoided.

Let

$$
\begin{aligned}
& \mathbf{f}_{k}\left(x^{\prime \prime}\right) \equiv \mathbf{m}_{k} f_{k}\left(x^{\prime \prime}\right) \\
& \mathbf{v}_{k}\left(x^{\prime \prime}\right) \equiv \partial_{x^{\prime \prime}} \mathbf{f}_{k}\left(x^{\prime \prime}\right)
\end{aligned}
$$

be two vector-valued functions. Representation (2.10) ensures that these functions are dimensionless and have the same orientation in $R^{3}$. With these functions Eq. (2.5) can be represented in a form of a six-dimensional system of ordinary differential equations of the first order:

$$
\frac{d}{d x^{\prime \prime}} \hat{\mathbf{z}}_{k}\left(x^{\prime \prime}\right)=\hat{\mathbf{G}} \cdot \hat{\mathbf{z}}_{k}\left(x^{\prime \prime}\right),
$$

where $\hat{\mathbf{z}}_{k}\left(x^{\prime \prime}\right)$ is a six-dimensional vector:

$$
\hat{\mathbf{z}}_{k}\left(x^{\prime \prime}\right)=\left(\begin{array}{c}
\mathbf{f}_{k}\left(x^{\prime \prime}\right) \\
\mathbf{v}_{k}\left(x^{\prime \prime}\right)
\end{array}\right)
$$

and $\hat{\mathbf{G}}$ is a $6 \times 6$ matrix:

$$
\hat{\mathbf{G}}=\left(\begin{array}{cc}
\mathbf{0} & \mathbf{I} \\
-\mathbf{A}_{1}^{-1} \cdot \mathbf{A}_{3} & -\mathbf{A}_{1}^{-1} \cdot\left(\mathbf{A}_{2}+\mathbf{A}_{2}{ }^{t}\right)
\end{array}\right) .
$$

In (2.13) $\mathbf{0}$ is the zero and $\mathbf{I}$ is the identity $3 \times 3$ matrix.

Proposition 2.1. Matrix $\hat{\mathbf{G}}$ is not singular, provided

$$
c^{2} \notin \frac{1}{\rho} \operatorname{Sp}\left(\mathbf{A}_{3}\right),
$$

where $\mathrm{Sp}$ denotes spectrum of the corresponding matrix.

Proof. The proof flows out from considering the determinant of matrix $\hat{\mathbf{G}}$ :

$$
\operatorname{det}(\hat{\mathbf{G}})=\operatorname{det}\left(\mathbf{A}_{1}^{-1} \cdot \mathbf{A}_{3}\right)=\operatorname{det}\left(\mathbf{A}_{1}^{-1}\right) \operatorname{det}\left(\mathbf{A}_{3}\right)
$$

and since matrix $\mathbf{A}_{1}$ is not singular, due to assuming positive definiteness of the elasticity tensor, condition (2.14) follows from expression (2.6) for $\mathbf{A}_{3}$. $\square$

As is known from the theory of ordinary differential equations [20, 21], the solution of Eq. (2.11) satisfying Cauchy's initial conditions at some value $x_{0}^{\prime \prime}$, can be represented in a form:

$$
\hat{\mathbf{z}}\left(x^{\prime \prime}\right)=\hat{\mathbf{R}}\left(x^{\prime \prime}-x_{0}^{\prime \prime}\right) \cdot \hat{\mathbf{z}}_{0},
$$

where $\hat{\mathbf{R}}$ is the fundamental matrix (fundamental system); see [21, Ch. IV, §2, n.4]:

$$
\hat{\mathbf{R}}\left(x^{\prime \prime}-x_{0}^{\prime \prime}\right)=e^{\hat{\mathbf{G}}\left(x^{\prime \prime}-x_{0}^{\prime \prime}\right)}
$$

and 


$$
\hat{\mathbf{z}}_{0} \equiv\left(\begin{array}{l}
\mathbf{f}_{0} \\
\mathbf{v}_{0}
\end{array}\right)
$$

is a 6-dimensional vector specifying the initial conditions at $x_{0}^{\prime \prime}$.

Remark 2.2. The solution in a form (2.16) is valid for any non-singular matrix $\hat{\mathbf{G}}$, regardless of its (semi)simplicity or non-semisimple degeneracy; see [20, Ch. IV, §5].

\section{Solution for a homogeneous layer}

\subsection{Traction-free plate}

Boundary conditions (1.1) can be rewritten in terms of convolution of a special $3 \times 6$ matrix composed of matrices (2.6) and vector $\hat{\mathbf{z}}\left(x^{\prime \prime}\right)$ :

$$
\left.\left.\left(\mathbf{A}_{2} ; \mathbf{A}_{1}\right) \cdot \hat{\mathbf{z}}\left(x^{\prime \prime}\right)\right|_{x^{\prime \prime}=-i r h} ^{x^{\prime \prime}=0} \equiv\left(\mathbf{A}_{1} \cdot \mathbf{v}\left(x^{\prime \prime}\right)+\mathbf{A}_{2} \cdot \mathbf{f}\left(x^{\prime \prime}\right)\right)\right|_{x^{\prime \prime}=-i r h} ^{x^{\prime \prime}=0}=0
$$

Substituting expression (2.16) for the vector $\hat{\mathbf{z}}\left(x^{\prime \prime}\right)$ into conditions (3.1) and taking $x_{0}^{\prime \prime}=0$, we arrive at the following equation:

$$
\left(\begin{array}{cc}
\mathbf{A}_{2} & \mathbf{A}_{1} \\
\mathbf{A}_{2} \cdot \mathbf{R}_{11}+\mathbf{A}_{1} \cdot \mathbf{R}_{21} & \mathbf{A}_{2} \cdot \mathbf{R}_{12}+\mathbf{A}_{1} \cdot \mathbf{R}_{22}
\end{array}\right) \cdot \hat{\mathbf{z}}_{0}=0,
$$

where $\mathbf{R}_{i j}$ are $3 \times 3$-matrix components of the fundamental matrix $\hat{\mathbf{R}}$ at $x^{\prime \prime}=-i r h$. Existence of a non-trivial 6-dimensional vector $\hat{\mathbf{z}}_{0}$ satisfying Eq. (3.2) is equivalent to vanishing the following determinant:

$$
\operatorname{det}\left(\begin{array}{cc}
\mathbf{A}_{2} & \mathbf{A}_{1} \\
\mathbf{A}_{2} \cdot \mathbf{R}_{11}+\mathbf{A}_{1} \cdot \mathbf{R}_{21} & \mathbf{A}_{2} \cdot \mathbf{R}_{12}+\mathbf{A}_{1} \cdot \mathbf{R}_{22}
\end{array}\right)=0
$$

The last equation can be rewritten in a form of vanishing the determinant of the third-order:

$$
\operatorname{det}\left(\mathbf{B}_{\text {left }} \cdot \hat{\mathbf{R}} \cdot \mathbf{B}^{\text {right }}\right)=0,(3.4)
$$

where

$$
\mathbf{B}_{\text {left }}=\left(\mathbf{A}_{2}, \mathbf{A}_{1}\right), \quad \mathbf{B}^{\text {right }}=\left(\begin{array}{c}
\mathbf{I} \\
-\mathbf{A}_{1}^{-1} \cdot \mathbf{A}_{2}
\end{array}\right) .
$$

Equation (3.4) is the secular equation for a traction-free plate.

\subsection{Clamped plate}

Boundary conditions (1.3) can be rewritten in terms of convolution of a special $3 \times 6$ matrix and vector $\hat{\mathbf{z}}\left(x^{\prime \prime}\right)$ :

$$
\left.\left.(\mathbf{I} ; \mathbf{0}) \cdot \hat{\mathbf{z}}\left(x^{\prime \prime}\right)\right|_{x^{\prime \prime}=-i r h} ^{x^{\prime \prime}=0} \equiv \mathbf{f}\left(x^{\prime \prime}\right)\right|_{x^{\prime \prime}=-i r h} ^{x^{\prime \prime}=0}=0 .
$$

Substituting expression (2.16) for the vector $\hat{\mathbf{z}}\left(x^{\prime \prime}\right)$ into conditions (3.6) and taking $x_{0}^{\prime \prime}=0$, we arrive at:

$$
\left(\begin{array}{cc}
\mathbf{I} & \mathbf{0} \\
\mathbf{R}_{11} & \mathbf{R}_{12}
\end{array}\right) \cdot \hat{\mathbf{z}}_{0}=0
$$


As before, components $\mathbf{R}_{i j}$ are $3 \times 3$-matrix components of the fundamental matrix $\hat{\mathbf{R}}$ at $x^{\prime \prime}=-i r h$. Existence of a non-trivial 6-dimensional vector $\hat{\mathbf{z}}_{0}$ satisfying Eq. (3.7) is equivalent to vanishing the determinant:

$$
\operatorname{det}\left(\begin{array}{cc}
\mathbf{I} & \mathbf{0} \\
\mathbf{R}_{11} & \mathbf{R}_{12}
\end{array}\right)=0 .
$$

The latter equation admits representation in a form of Eq. (3.4) with

$$
\mathbf{B}_{\text {left }}=(\mathbf{I}, \mathbf{0}), \quad \mathbf{B}^{\text {right }}=\left(\begin{array}{l}
\mathbf{0} \\
\mathbf{I}
\end{array}\right) \text {. }
$$

\subsection{Plate with mixed boundary conditions}

By analogy with Eqs. (3.2) and (3.7), boundary conditions (1.4) yield:

$$
\left(\begin{array}{cc}
\mathbf{A}_{2} & \mathbf{A}_{1} \\
\mathbf{R}_{11} & \mathbf{R}_{12}
\end{array}\right) \cdot \hat{\mathbf{z}}_{0}=0
$$

It can be shown that Eq. (3.10) can be represented in a form (3.4) with

$$
\mathbf{B}_{\text {left }}=(\mathbf{I}, \mathbf{0}), \quad \mathbf{B}^{\text {right }}=\left(\begin{array}{c}
\mathbf{I} \\
-\mathbf{A}_{1}^{-1} \cdot \mathbf{A}_{2}
\end{array}\right) .
$$

Remark 3.1. a) Thus, dispersion relations for Lamb waves propagating in a single layered plate with the considered three types of boundary conditions, satisfy the unique secular equation (3.4) with different matrices $\mathbf{B}_{\text {left }}, \mathbf{B}^{\text {right }}$.

b) Problems and algorithms for numerical evaluation of a matrix exponential are discussed in [22 - 25].

\section{Solution for a layered plate}

Let a multilayered plate consists of $N$-layers, Fig. 2. The lower index $n$ will be referred to the corresponding layer.

Assuming ideal mechanical contact at the interfaces and taking into account nondegeneracy of matrix $\mathbf{A}_{1}$, Eqs. (1.5) can be replaced by equations of continuity of the solution of Eq. (2.16) across the interfaces:

$$
\hat{\mathbf{z}}_{n+1}(0)=\hat{\mathbf{z}}_{n}\left(-i r h_{n}\right), \quad n=1, \ldots, N-1 .
$$

Equations (4.1) are written in local coordinate systems with origin at the upper boundary of each layer. Conditions (4.1) allow us to represent $\hat{\mathbf{z}}_{N}\left(-i r h_{N}\right)$ in terms of vector $\hat{\mathbf{z}}_{0}$, which specifies initial conditions at the upper boundary, and convolution of the fundamental matrices:

where

$$
\hat{\mathbf{z}}_{N}\left(-i r h_{N}\right)=\hat{\mathbf{R}} \cdot \hat{\mathbf{z}}_{0}
$$

$$
\hat{\mathbf{R}}=e^{-i r \hat{\mathbf{G}}_{N} h_{N}} \cdot \ldots \cdot e^{-i r \hat{\mathbf{G}}_{1} h_{1}}
$$

Remark 4.1. a) Matrix $\hat{\mathbf{R}}$ plays role of the transfer matrix, as it transfers initial values $\hat{\mathbf{z}}_{0}$ at $x^{\prime \prime}=0$ to $\hat{\mathbf{z}}_{N}\left(-i r h_{N}\right)$. Within framework of the 3-dimensional formalism applied to surface wave analysis, the transfer matrices were introduced in [26, 27]; see also [28 - 30], where different modifications of the transfer matrix method are suggested to improve numerical stability.

b) It should be noted that generally 


$$
e^{-i r \hat{\mathbf{G}}_{N} h_{N}} \cdot \ldots \cdot e^{-i r \hat{\mathbf{G}}_{1} h_{1}} \neq e^{-i r\left(\sum \hat{\mathbf{G}}_{n} h_{n}\right)}
$$

because of the non-commutative property of matrices $\hat{\mathbf{G}}_{n}$.

c) However, matrices $\hat{\mathbf{G}}_{n}$ and $\exp \left(\hat{\mathbf{G}}_{n}\right)$ commute, whatever matrix $\hat{\mathbf{G}}_{n}$ may be. Now, by applying boundary conditions to Eq. (4.2), the secular equation for dispersion curves in a multilayered plate takes form (3.4) with matrix $\hat{\mathbf{R}}$ defined by (4.3), and the auxiliary matrices $\mathbf{B}_{\text {left }}, \mathbf{B}^{\text {right }}$ defined by (3.5) for a traction-free plate, by (3.9) for a clamped plate, and by (3.11) for a plate with mixed boundary conditions.

\section{Conclusions}

The Cauchy six-dimensional formalism is developed for analysing propagation of Lamb waves in anisotropic multilayered plates. Comparison with the formalism, known as the 3D formalism, is done, revealing the anticipated benefits of the Cauchy formalism in respect of its ability to construct the closed form dispersion equation and the corresponding solution for dispersion curves.

The author (SVK) thanks the Russian Foundation for Basic Research (Grants 17-08-00311; 18-5841001; 19-01-00100) and the Programs 27, 32, 55 of the Presidium of RAS for partial financial support.

\section{References}

1. Lamb H 1917 On waves in an elastic plate, Proc. Roy. Soc. A93 114-28

2. Strutt J W (Lord Rayleigh) 1885 On wave propagating along the plane surface of an elastic solid Proc. London Math. Soc. 17 4-11

3. Farnell G W 1970 Properties of elastic surface waves Phys. Acoust. 6 109-66

4. Chadwick P and Smith G D 1977 Foundations of the theory of surface waves in anisotropic elastic materials Acad. Press, N.Y. Advances in Applied Mechanics $17303-$ 76 ,

5. Ting T C T and Barnett D M 1997 Classifications of surface waves in anisotropic elastic materials Wave Motion 26 207-18

6. Mase G T and Johnson G C 1987 An acoustic theory for surface waves in anisotropic media J. Appl. Mech. 54, 127 -35

7. Lin W and Keer L M 1992 A study of Lamb waves in anisotropic plates J. Acoust. Soc. Amer. 92, 888-94

8. Kuznetsov S V 2002 Subsonic Lamb waves in anisotropic plates Quart. Appl. Math. 60, 577-587

9. Nayfen A H 1991 The general problem of elastic waves propagation in multilayered anisotropic media J. Acoust. Soc. Am. $891521-31$

10. Nayfen A H 1989 The propagation of horizontally polarized shear waves in multilayered anisotropic media, J. Acoust. Soc. Am. $862007-12$

11. Guo $\mathrm{N}$ and Cawley $\mathrm{P} 1993$, Lamb wave propagation in composite laminates and its relationship with acousto-ultrasonics NDT \& E Int. 26 75-84

12. Chimenti D E 1994 Lamb waves in microstructured plates Ultrasonics 32 255-60

13. Lowe M J S 1995 Matrix techniques for modeling ultrasonic waves in multilayered media, IEEE Transactions on Ultrasonics, Ferroelectrics, and Frequency Control 42 $525-42$

14. Ting T C T and Chadwick P 1988, Harmonic waves in periodically layered anisotropic elastic composites ASME Symposium Wave Propagation in Structural Composites 90 67-9 
15. Braga A M and Hermann G 1992, Floquet waves in anisotropic periodically layered composites, J. Acoust. Soc. Am. $911211-27$

16. Stroh A N 1962, Steady state problems in anisotropic elasticity J. Math. Phys. 4177 103

17. Kuznetsov S V 2003 , Surface waves of Non-Rayleigh type Quart. Appl. Math. 61 58792

18. Kuznetsov S V 2004, Love waves in stratified monoclinic media Quart. Appl. Math. 62 $749-66$

19. Djeran-Maigre I and Kuznetsov S V 2008 Solitary SH waves in two-layered tractionfree plates Comptes Rendus Mécanique 336 102-7

20. Hartman P 1964 Ordinary Differential Equations (N. Y : Wiley) p 612

21. Bourbaki N 1965, Elements de Mathematique. Fonctions d'une Variable Reelle (Theorie Elementaire), (Paris: Hermann) p 329

22. Leonard I E 1996 The matrix exponential SIAM Rev. 38 507-12

23. Liz E 1998 A note on the matrix exponential SIAM Rev. 40 700-2

24. Harris W A, Fillmore J P, and Smith D R 2001 Matrix exponential-another approach SIAM Rev. 43 694-706

25.Zanna A and Munthe- Kaas H Z 2002 Generalized polar decompositions for the approximation of the matrix exponential SIAM J. Matrix Anal. Appl. 23 840-62

26. Thomson W T 1950 Transmission of elastic waves through a stratified solid medium $J$. Appl. Phys. $2189-93$

27. Haskell N A 1953 Dispersion of surface waves on multilayered media Bull. Seism. Soc. Am. $4317-34$

28. Wang L and Rokhlin S I 2001 Stable reformulation of transfer matrix method for wave propagation in layered anisotropic media Ultrasonics 39 413-24

29. Wang L and Rokhlin S I 2004 Modeling of wave propagation in layered piezoelectric media by a recursive asymptotic matrix method IEEE Trans. Ultrason. Ferroelectr. Freq. Control 51 1060-71

30. Djeran-Maigre I and Kuznetsov S V 2014 Velocities, dispersion, and energy of SHwaves in anisotropic laminated plates Acoustical Physics 60 200-7 